\title{
Towards closing the window of primordial black holes as dark matter: the case of large clustering
}

\author{
Torsten Bringmann* \\ Department of Physics, University of Oslo, Box 1048, N-0371 Oslo, Norway

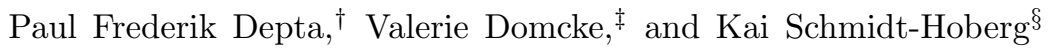 \\ Deutsches Elektronen-Synchrotron DESY, Notkestraße 85, D-22607 Hamburg, Germany
}

\begin{abstract}
The idea of dark matter in the form of primordial black holes has seen a recent revival triggered by the LIGO detection of gravitational waves from binary black hole mergers. In this context, it has been argued that a large initial clustering of primordial black holes can help alleviate the strong constraints on this scenario. In this letter, we show that on the contrary, with large initial clustering the problem is exacerbated and constraints on primordial black hole dark matter become overwhelmingly strong.
\end{abstract}

Introduction. - Soon after realising that black holes (BHs) could form in the early radiation-dominated universe [1] from the gravitational collapse of large density fluctuations, it was pointed out that such objects may even contribute appreciably to the total matter density [4. An obvious question is therefore whether these primordial black holes (PBHs) could explain all of the cosmologically observed dark matter (DM), see Refs. [5, 6] for recent reviews. This idea has seen greatly renewed interest [7 11] after the discovery of binary mergers by the Advanced Laser Interferometer Gravitational-Wave Observatory (LIGO) [12 15], proving the existence of $\mathcal{O}\left(10 M_{\odot}\right) \mathrm{BHs}$ with so far unclear origin.

Constraints on the allowed DM fraction $f_{\mathrm{PBH}}$ of $\mathrm{PBHs}$ derive from a large number of observations and have been explored for a vast range of mass scales, see Refs. [5, 6, 10] for an overview. While there seems to be a broad consensus that $f_{\mathrm{PBH}} \sim 1$ is essentially excluded for black hole masses $m_{\mathrm{PBH}} \gtrsim 10^{-10} M_{\odot}$ when assuming a homogeneously distributed population of $\mathrm{PBH}$ with a single mass, this general picture changes when either of these conditions is not met. Intriguingly, this also opens the window of PBH masses consistent with the LIGO observations by circumventing the stringent constraints from microlensing and from the cosmic microwave background (CMB) [16] (see however [17, 18]).

Clustered PBH distributions have been argued to arise generically in Refs. [19, 20, possibly explaining the existence of super-massive BHs [21, 22. Only recently it was realized that significant clustering is in fact not expected for Gaussian primordial fluctuations [23, 24], as predicted by vanilla models of cosmic inflation. However, highly clustered PBH distributions could still plausibly arise, e.g., in the presence of sizeable primordial non-Gaussianities [25] or from the collapse of domain walls [26. In this Letter we choose to be agnostic about the possible origin of large $\mathrm{PBH}$ clustering. Instead we demonstrate that in such a situation $f_{\mathrm{PBH}} \sim 1$ is in fact still excluded over a wide mass-range, thereby closing this possibly last loop-hole for all DM consisting of PBHs with masses larger than $10^{-10} M_{\odot}$.

The initial clustering of PBHs is indeed a key parameter to understanding the phenomenology of PBH DM, affecting merger rates [27, 28], the subsequent structure formation [29], and the interpretation of observational bounds [16]. Here we take a pragmatic and phenomenological approach by parametrising the clustering as a constant, free parameter on the scales of interest. We point out that, for the large $\mathrm{PBH}$ clustering discussed in the literature, the expected merger rates easily exceed one per binary and Hubble time. We demonstrate that multiple subsequent mergers severely constrain PBH DM as a possible explanation of the LIGO events because of i) the expected (as compared to observed) merger rate, ii) the impact of the additional radiation component in gravitational waves (GWs) on both $\mathrm{CMB}$ and large-scale structure observations, and iii) a present-day stochastic GW background (SGWB) exceeding the sensitivities of current ground- (or future space-) based observatories.

This Letter is organised as follows. We start by describing the GW spectrum and energy density from cosmological PBH mergers, before discussing how the merger rate critically depends on the initial PBH clustering. We then introduce a cascading merger scenario to capture the effects of large clustering, and hence high merger rates. We derive the resulting contributions to the stochastic GW background and the relativistic energy density in GWs, using the cosmological parameters from Ref. 30] whenever relevant. Along with the actual event rate observed by LIGO, we use this to place constraints on $f_{\mathrm{PBH}}$. In the accompanying material [31] we show that deviating from our assumptions in the main text does not qualitatively change our results.

Gravitational waves from merging black holes.- Coalescing binary BHs emit GWs with a characteristic spectrum $\mathrm{d} E_{\mathrm{GW}} / \mathrm{d} \nu$, with a total energy that makes up a significant fraction of the rest mass [32. For BHs with identical masses and negligible spins, e.g., one expects $E_{\mathrm{GW}} / M_{2 \mathrm{BH}} \simeq 5 \%$, where $M_{2 \mathrm{BH}}$ is the initial mass of the system. For the ten events observed so far by LIGO, this 
number ranges between $3.3 \%$ and $5.4 \%$ [33].

In the following we will study the cumulative effect of all mergers throughout the cosmological evolution. The resulting present energy density per logarithmic frequency interval is conventionally expressed in units of the critical density, $\rho_{c}=3 H_{0}^{2} /(8 \pi G)$, and computed as [34, 35]

$$
\Omega_{\mathrm{GW}}(\nu)=\frac{1}{\rho_{c}} \int_{0}^{\infty} \mathrm{d} \tilde{z} \int \mathrm{d} R(\tilde{z}) \frac{\nu}{(1+\tilde{z}) H(\tilde{z})} \frac{\mathrm{d} E_{\mathrm{GW}}}{\mathrm{d} \nu_{s}} .
$$

Here, the merger rate is denoted as $R(z)$, where $z$ is the cosmological redshift, $H$ is the Hubble rate, and the observed frequency $\nu$ corresponds to an emission frequency of $\nu_{s}=\nu(1+z)$. The total energy density in gravitational waves at any given redshift $z \equiv a-1$ is therefore

$$
\frac{\rho_{\mathrm{GW}}(z)}{(1+z)^{4}}=\int_{z}^{\infty} \mathrm{d} \tilde{z} \int \mathrm{d} \nu \int \mathrm{d} R(\tilde{z}) \frac{\mathrm{d} E_{\mathrm{GW}} / \mathrm{d} \nu}{(1+\tilde{z})^{2} H(\tilde{z})} .
$$

Covariant conservation of energy implies that the mass density in $\mathrm{PBHs}$ must correspondingly decrease as $a^{-3} \mathrm{~d}\left(a^{3} \rho_{\mathrm{PBH}}\right)=-a^{-4} \mathrm{~d}\left(a^{4} \rho_{\mathrm{GW}}\right)$ [36], which gives

$$
\frac{\rho_{\mathrm{PBH}}(z)}{(1+z)^{3}}=C-\int_{z}^{\infty} \mathrm{d} \tilde{z} \int \mathrm{d} \nu \int \mathrm{d} R(\tilde{z}) \frac{\mathrm{d} E_{\mathrm{GW}} / \mathrm{d} \nu}{(1+\tilde{z}) H(\tilde{z})} .
$$

We fix the integration constant $C$ such that $f_{\mathrm{PBH}} \equiv\left(\rho_{\mathrm{PBH}} / \rho_{\mathrm{DM}}\right)_{z_{\mathrm{CMB}}}$ is the $\mathrm{PBH}$ fraction at $z=z_{\mathrm{CMB}} \simeq 1100$. For the spectrum $\mathrm{d} E_{\mathrm{GW}} / \mathrm{d} \nu$ we use commonly adopted fitting formulae [37 40].

Merger rates and clustering. - In the early universe, PBH binary formation starts once the Newtonian force between two initial PBHs overcomes the Hubble flow, with a nearby third PBH providing the angular momentum necessary to prevent a head-on collision [9, 41, 42]. Later, peculiar velocities may be too large for this to happen; instead, binary formation can be triggered by the energy loss in GWs during close encounters of two PBHs [43, 44. For the parameter combinations of interest to us, though, the rate associated to the first formation mechanism largely exceeds that for the second, even for binaries merging only today [27. Once formed, these binary systems survive until they merge, largely unaffected by the evolution of the surrounding Universe [45].

A crucial input for calculating those merger rates is the initial clustering of PBHs. Phenomenologically, this can be described in terms of an idealised two-point correlation function $\xi_{\mathrm{PBH}}(r)$ that is constant at scales relevant for the formation of PBH binaries [27:

$$
1+\xi_{\mathrm{PBH}}(r) \approx \delta_{\mathrm{dc}}=\text { const. },
$$

where $\delta_{\mathrm{dc}}$ describes the local density contrast, evaluated at the time when the two BHs decouple from the Hubble expansion. A perfectly homogeneous PBH distribution corresponds to $\delta_{\mathrm{dc}}=1$, while a highly clustered PBH distribution is described by $\delta_{\mathrm{dc}} \gg 1$. Values of $\delta_{\mathrm{dc}} \gtrsim 10^{5}$ are particularly interesting, as they are required to circumvent the tight constraints on PBH DM from microlensing and the CMB [16. Furthermore, constraints arising from the conversion of PBH DM into gravitational radiation are alleviated if PBH mergers occur only at high redshift, which was demonstrated to happen for $\delta_{\mathrm{dc}} \gtrsim 10^{4}$ assuming only a single merger step [27.

We will see that a highly clustered initial PBH population not only leads to a more efficient formation of binaries but also to a significantly enhanced merger rate, during the whole cosmological evolution until today. These findings crucially extend previous results in the literature (see e.g. 27, 28]), which consider the impact of clustering only on a single merger step.

Cascading black hole merger events. - We thus need to improve these scenarios by allowing for subsequent merger steps, i.e. binary mergers of systems of previously merged PBHs. For simplicity, we model the PBHS distribution as an initially monochromatic mass distribution peaked at $m_{0}$ and assume that the PBH masses in merger step $j$ are given by

$$
m_{j}=2 m_{j-1}-E_{\mathrm{GW}}\left(m_{j-1}\right) \sim 1.9^{j} m_{0} .
$$

The impact of more realistic, extended initial mass functions is discussed in the accompanying material, where we demonstrate that our key results are not affected by this choice. The average PBH number density $n_{j}=\rho_{\mathrm{PBH}, \infty} /\left(2^{j} m_{0}\right)$ is locally enhanced by a factor of $1+\xi(r) \approx \delta_{\mathrm{dc}, j}$, where $\rho_{\mathrm{PBH}, \infty}$ denotes the initial $\mathrm{PBH}$ density and we model the decrease of the PBH clustering as $\delta_{\mathrm{dc}, j} \simeq 2^{-j} \delta_{\mathrm{dc}, 0}$. The details of this decrease will in general depend on the exact form of the two-point correlation function; as argued in the accompanying material, the specific choice above leads to rather conservative limits on the fraction of PBH DM.

The merger rate of the $j$ th merger step is given by

$$
\begin{aligned}
R_{j}(t)= & \int_{0}^{\tilde{x}} \mathrm{~d} x \int_{\tilde{x}}^{\infty} \mathrm{d} y \frac{\partial^{2} n_{3, j}(x, y)}{\partial x \partial y} \\
& \delta\left(t-\tau\left(x, y, m_{j}\right)-\max \left(t_{\mathrm{dc}, j}(x), t_{\mathrm{form}}\right)\right),
\end{aligned}
$$

where $x$ and $y$ denote the comoving distances from a given $\mathrm{PBH}$ to the nearest and next-to-nearest $\mathrm{PBH}$, respectively, and the number density of $\mathrm{PBH}$ triples $n_{3, j}(x, y)$ is given by 27 .

$$
\mathrm{d} n_{3, j}(x, y)=\frac{n_{j}}{2} \mathrm{e}^{-\frac{4 \pi}{3} y^{3} n_{j} \delta_{\mathrm{dc}, j}}\left(4 \pi n_{j} \delta_{\mathrm{dc}, j}\right)^{2} x^{2} y^{2} \mathrm{~d} x \mathrm{~d} y .
$$

The delta distribution in Eq. (6) ensures that the coalescence time $\tau$ [46],

$$
\tau\left(x, y, m_{j}\right)=\tilde{\tau}_{j}\left(x / \tilde{x}_{j}\right)^{37}\left(y / \tilde{x}_{j}\right)^{-21},
$$

with

$$
\tilde{\tau}_{j}=\frac{3 a_{\mathrm{eq}}^{4} \tilde{x}_{j}^{4}}{170\left(G m_{j}\right)^{3}}, \quad \tilde{x}_{j}^{3}=\frac{3}{4 \pi} \frac{2 m_{j}}{a_{\mathrm{eq}}^{3} \rho_{\mathrm{eq}}}
$$




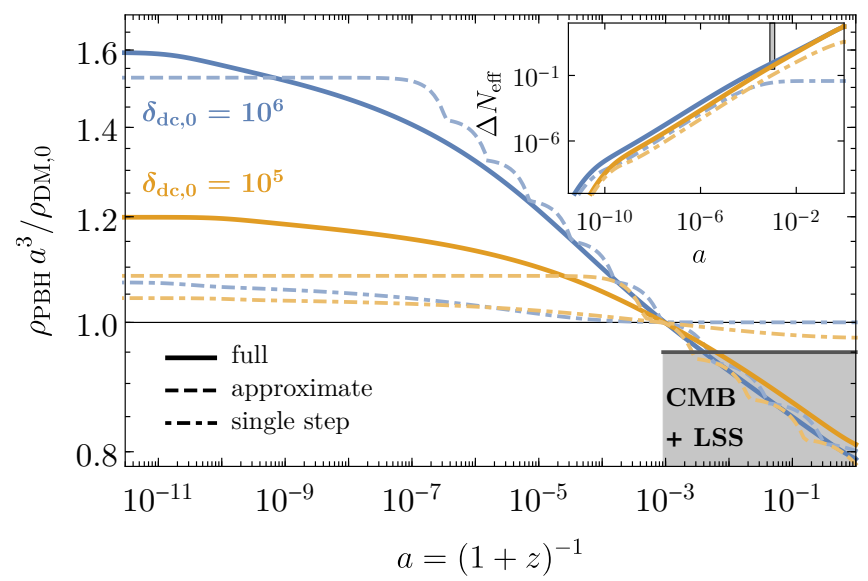

FIG. 1. Conversion of PBH energy density (main panel) into gravitational wave radiation (in terms of a corresponding number $\Delta N_{\text {eff }}$ of additional neutrinos, top right inlet) for $m_{0}=1 M_{\odot}, f_{\mathrm{PBH}}=1$, and different theoretical treatments. In grey, we indicate regions excluded by cosmology [36].

is measured from when the PBHs are both formed $\left(t_{\text {form }}\right)$ and decoupled from the Hubble flow $\left(t_{\mathrm{dc}, j}\right)$. PBHs form almost immediately after the corresponding density perturbations enter the Hubble horizon, with a mass $m_{0}$ equalling the total energy within the horizon at that time, and the decoupling from the Hubble flow occurs when the gravitational attraction overcomes the Hubble expansion:

$$
t_{\text {form }}=G m_{0}, \quad t_{\mathrm{dc}, j}=\left(\frac{16 \pi G}{3} \rho_{\mathrm{eq}}\right)^{-1 / 2}\left(\frac{x}{\tilde{x}_{j}}\right)^{6} .
$$

The subscript 'eq' above refers to matter radiation equality. The merger rates $R_{j}$ in Eq. (6) are connected to the differential one employed in Eqs. (1)-(3) via

$$
\mathrm{d} R(\tilde{z})=\sum_{j} R_{j}(t(\tilde{z})) \delta\left(m-m_{j}\right) \mathrm{d} m .
$$

To recap, we consider a scenario of subsequent equalmass mergers with a corresponding shift in the mass distribution and local density contrast in each merger step. Let us stress that even though there are characteristic time-scales implied by the merger rates, we allow PBHs of given mass $m_{j}$ to merge at any time between the decoupling for the $j$ th step (as long as $t_{\mathrm{dc}, j}>t_{\text {form }}$ ) and today. For rare very early mergers this may lead to situations where coalescence in our model begins when, in reality, instead of the eventually merging two $\mathrm{PBHs}$ a preceding set of smaller PBHs is present; in this case, we slightly underestimate the actual amount of emitted GWs. We further introduce an approximate scenario to better visualise the individual merger steps, adopting rates $R_{j}$ that are zero before $\max \left(t_{\mathrm{dc}, j}, t_{\text {form }}\right)$, constant until the average coalescence time has passed (with average values for $x$ and $y$ ), and zero afterwards.

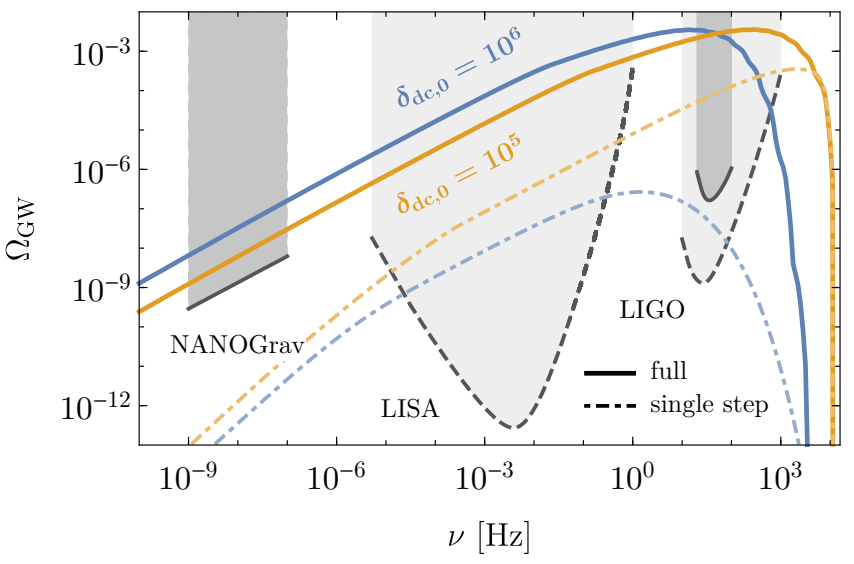

FIG. 2. GW density parameter per logarithmic frequency interval for $m_{0}=1 M_{\odot}, f_{\mathrm{PBH}}=1$, and different theoretical treatments. In grey, we indicate present (solid lower lines) and projected (dashed lower lines) constraints from NANOGrav [4], LISA 49, and LIGO 50] (present O1 and projected design constraint).

In Fig. 1 we show the decrease of the PBH energy density in our merger scenario (solid lines) as well as the corresponding increase in gravitational wave radiation (top right inlet). Overall, the agreement between the full rates, as computed from Eq. (6), and the simple approximate scenario mentioned above (dashed lines) is fairly good. In particular we note the sizeable equidistant spacing of the merger steps, justifying the assumption of a hierarchical merging scenario. The dash-dotted curves represent the common assumption of a single merger step. For larger values of $\delta_{\mathrm{dc}, 0}$, this merger step occurs earlier [27, leading to the tempting conclusion that bounds on the $\mathrm{GW}$ production can be evaded since the produced radiation is highly red-shifted. As we discuss below, this conclusion clearly no longer holds once multiple merger steps are taken into account.

Cosmological bounds. - The conversion of PBH DM into GW radiation modifies the standard cosmological evolution and is constrained by CMB and large scale structure (LSS) observations [36. This can be roughly split into i) an upper bound on the effective number of neutrino species at the time of the CMB, $\Delta N_{\text {eff }}\left(z_{\mathrm{CMB}}\right) \lesssim$ 0.3 [30, indicated by the grey area in the inlet of Fig. 1. and ii) the amount of DM converted into invisible ('dark') radiation at later times. From Fig. 6 in Ref. 36] one can deduce that not more than $\sim 5 \%$ of DM can be converted into dark radiation after the CMB epoch, irrespective of the precise time-dependence of this conversion (and consistent with the $4.2 \%$ found for the case of decaying DM [47]). For simplicity, and because other constraints turn out to be stronger, we conservatively adopt this bound of $5 \%$ in our analysis (indicated by the grey region in the bottom right of Fig. 11). 


\begin{tabular}{c|cccccccc}
$m / M_{\odot}$ & 0.2 & 1 & 10 & 20 & 40 & 100 & 200 & 300 \\
$R \mathrm{Gpc}^{3} \mathrm{yr}$ & $10^{6}$ & $1.9 \cdot 10^{4}$ & 330 & 77 & 15 & 2 & 5 & 20
\end{tabular}

TABLE I. 90\% CL upper limits on merger rates in the late universe, taken from Refs. 52 55].

The stochastic gravitational wave background.With the cumulative merger rate described above, it is also straightforward to compute the resulting SGWB as given in Eq. (1). In Fig. 2, we illustrate the predictions for an initial PBH mass of $1 M_{\odot}$ and different initial clusterings $\delta_{\mathrm{dc}, 0}=10^{5}$ and $\delta_{\mathrm{dc}, 0}=10^{6}$. The GW spectrum is dominated by late time mergers $(z \lesssim 10)$, since earlier GW emission is highly diluted by cosmic expansion. Larger clustering implies that most of these late mergers are associated with heavier BHs, which emit GWs with lower frequencies. For the parameter example of Fig. 2 we find that the mergers occurring at $z=0$ have typically undergone $4(9)$ previous mergers for $\delta_{\mathrm{dc}, 0}=10^{5}\left(10^{6}\right)$, resulting in a PBH mass that is larger by a factor of about 20 in the latter case. Since the frequency of the emitted GWs roughly scales as $\nu \sim 1 / M_{2 \mathrm{BH}}$, cf. [27, 51, this explains the shift between the two solid lines in Fig. 2 . Moreover, the merger cascade described above leads to a mild broadening of the high frequency peak. At low frequencies, the $\Omega_{\mathrm{GW}} \sim \nu^{2 / 3}$ scaling indicates the early inspiral phase of the $\mathrm{BH}$ binaries [38].

For comparison, the dash-dotted curves show the predictions for a single merger step, where the main effect of large clustering is to shift the merging time to high redshift, strongly suppressing the GW spectrum. However, as Fig. 2 demonstrates, later mergers completely change the picture, leading to a large contribution to the SGWB. The grey contours, finally, indicate the power-law integrated sensitivity curves of LIGO [50] and the pulsar timing array NANOGrav [48, as well as the planned spacebased LISA [49] observatory.

Observed merger rate. - The LIGO/VIRGO observations strongly constrain the merger rate of $\mathrm{PBHs}$ with masses between 0.2 and $300 M_{\odot}$ [52 [55]. Interpolating linearly between the limiting rates stated in Tab. I. and comparing this to the calculated $R_{j}(z=0)$, allows us to derive an upper bound on $f_{\mathrm{PBH}}$. Starting with an initially monochromatic mass function, we would not expect to reproduce the $\mathrm{BH}$ mass distribution observed by LIGO. However, requiring to reproduce the total observed merger rate $\left(12-213 \mathrm{Gpc}^{-3} \mathrm{yr}^{-1}\right.$ [15]) with $\mathrm{PBH}$ mergers in the sensitivity band of LIGO $\left(7-50 M_{\odot}\right)$, we obtain a (very conservative) range in $m_{0}$ compatible with the total merger rate observed by LIGO.

Results. - In the left panel of Fig. 3, we summarise the resulting constraints on the allowed fraction of DM in PBHs for large initial clustering, $\delta_{\mathrm{dc}, 0}=10^{6}$, as a function of the initial PBH mass $m_{0}$ (the shaded regions are excluded). For reference, we also indicate contour lines with the present, rate-averaged PBH mass $m_{\text {avg. }}$. We depict as blue-green and orange curves, respectively, the cosmological constraints 36 indicated as grey shaded areas in Fig. 1. The blue solid line shows the merger rate constraint; we note that it extends to average $\mathrm{PBH}$ masses well below the LIGO/VIRGO limit because a small fraction of PBHs will still satisfy $m_{\mathrm{PBH}}>0.2 M_{\odot}$ after many merger steps. The remaining lines, finally, correspond to the SGWB constraints from NANOGrav (green) and LIGO (purple) indicated in Fig. 2. The upcoming space-based LISA experiment (red, dashed) may severely tighten these constraints.

In the right panel of Fig. 3 , we show our combined results on $f_{\mathrm{PBH}}$, illustrating that larger values of the clustering parameter $\delta_{\mathrm{dc}, 0}$ in fact lead to tighter constraints. For comparison, the dash-dotted lines indicate the much weaker constraints obtained when taking into account only a single merger step. The arrows indicate the range for $m_{0}$, where for a suitable $f_{\mathrm{PBH}}$ the total present merger rate is consistent with all observed LIGO events being caused by PBH mergers.

Discussion. - We made a number of simplifying assumptions when modelling the PBH merger history, which can impact the details of the limits summarized in Fig. 3. As we argue below, however, our main result that a large clustering leads to a tightening of existing limits is robust. This implies, in particular, that the BHs observed by LIGO cannot be part of a PBH population comprising all of the DM.

To start with, we have throughout assumed a monochromatic initial PBH mass distribution. While a full investigation of extended mass distributions is beyond the scope of this work, we have checked explicitly that a log-normal initial mass distribution does not qualitatively change the merger history [31] (nor the SGWB constraints [27]). Another crucial input was our assumption that the local $\mathrm{PBH}$ number density contrast decreases as $\delta_{\mathrm{dc}, \mathrm{j}}=0.5^{j} \delta_{\mathrm{dc}, 0}$. If the density contrast decreases less rapidly (which in fact is a more realistic assumption in many situations) the constraints soon become significantly stronger even for less 'extreme' initial values of $\delta_{\mathrm{dc}}$ 31. As we further argue in 31, it is also a well justified approximation to neglect the effect of GW recoils of newly formed binaries.

Conclusions. - If PBHs are not homogeneously distributed in the Universe but highly clustered, existing bounds on their abundance must be re-interpreted. Here we have demonstrated that the resulting limits are not weakened, as claimed previously, but instead strengthened because subsequent merger steps would dominate the SGWB. Taking into account constraints from cosmology and direct GW searches, we find that for $\delta_{\mathrm{dc}, 0}>10^{4}$ the case of pure PBH DM is firmly excluded in the entire range of initial PBH masses between $10^{-5} M_{\odot}$ and $100 M_{\odot}$. For slightly less conservative assumptions about 

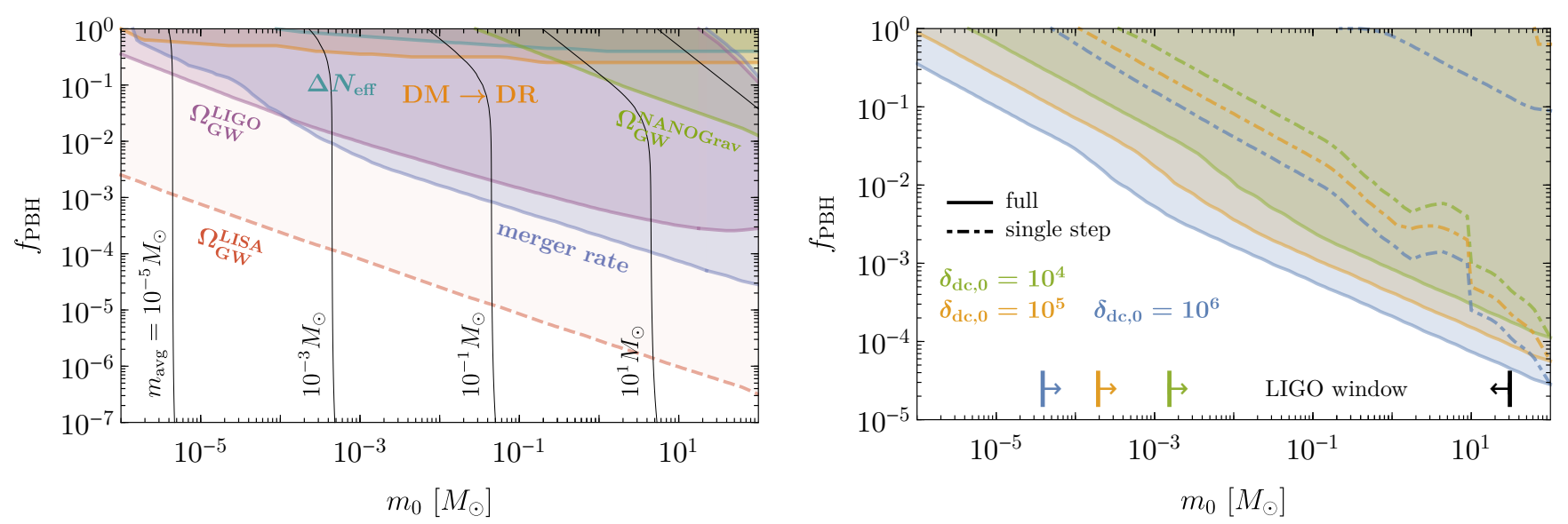

FIG. 3. Left. Constraints on the allowed fraction $f_{\mathrm{PBH}}$ of $\mathrm{PBH} \mathrm{DM}$ as a function of the initial $\mathrm{PBH}$ mass $m_{0}$ for large clustering $\left(\delta_{\mathrm{dc}, 0}=10^{6}\right)$ in the merger cascade scenario. The thinner black lines indicate contours of the rate-averaged PBH mass $m_{\mathrm{avg}}=\left(\sum_{j} R_{j} m_{j}\right) / \sum_{j} R_{j}$ at $z=0$. Right. Combined constraints on $f_{\mathrm{PBH}}$ for different clustering parameters $\delta_{\mathrm{dc}, 0}$.

the decrease of $\delta_{\mathrm{dc}}$ in subsequent merger steps, this even holds for much smaller initial density contrasts 31. We note that outside this mass range bounds are also very strong [10, which essentially left this interval as one out of only two realistic options for explaining all DM in terms of PBHs (the second one arises for much lighter PBHs of $\left.10^{-16} \lesssim m_{\mathrm{PBH}} / M_{\odot} \lesssim 10^{-11}[56]\right)$.

Acknowledgements. - We thank Thomas Konstandin for useful comments on the manuscript, Florian Kühnel and Hardi Veermäe for relevant discussions and Cole Miller for encouraging us to spell out why the gravitational wave recoil does not significantly affect our analysis. We would also like to thank the anonymous referees for pertinent comments. This work is supported by the German Science Foundation (DFG) under the Collaborative Research Center (SFB) 676 Particles, Strings and the Early Universe as well as the ERC Starting Grant 'NewAve' (638528). TB wishes to thank McGill university, where part of this manuscript was completed, for support and hospitality. This research was supported in part by Perimeter Institute for Theoretical Physics. Research at Perimeter Institute is supported by the Government of Canada through the Department of Innovation, Science and Economic Development and by the Province of Ontario through the Ministry of Research, Innovation and Science.

\section{EXTENDED MASS DISTRIBUTIONS}

In the main text, we focused for simplicity on an initially monochromatic PBH mass distribution. Realistic models of PBH formation however predict extended mass functions. For example, if the scalar perturbations which collapse into PBHs are formed during single-field inflation, then the characteristic time-scale governing the width of any peaked feature in the scalar power spectrum is the Hubble time. Consequently, the resulting $\mathrm{PBH}$ mass distribution is expected to be rather broad, since the range of enhanced length scales is exponentially sensitive to this time-scale [57] (though notable exceptions exist 58 61]). More generally, even if the spectrum of enhanced scalar perturbations is essentially monochromatic, the process of collapsing the initial perturbations into PBHs obeys a critical scaling relation, implying a finite width for the resulting PBH mass spectrum [5]. Here we discuss how the results in the main text are affected by relaxing the assumptions of an initially monochromatic PBH mass distribution.

To model an extended PBH mass function, we consider the lognormal mass distribution

$$
\psi_{\log }(m)=\frac{f_{\mathrm{PBH}, \infty}}{\sqrt{2 \pi} \sigma m} \exp \left(\frac{\log ^{2}\left(m / m_{0}\right)}{2 \sigma^{2}}\right),
$$

where $\sigma$ encodes the width of the distribution centered around the reference scale $m_{0}$. This distribution is normalized so that $f_{\mathrm{PBH}, \infty}=\int_{0}^{\infty} \mathrm{d} m \psi(m)$. Note that for computational simplicity, we fix the $\mathrm{PBH}$ fraction of DM at $z \rightarrow \infty$ in this chapter as indicated by the subscript $\infty$. In our scenario of multiple mergers, the strongest constraints on the PBH abundance arise from the bounds on the SGWB and from the observed merger rate in LIGO, see Fig. 3. Consequently we will focus on the impact of the distribution (12) on these two quantities, noting that 


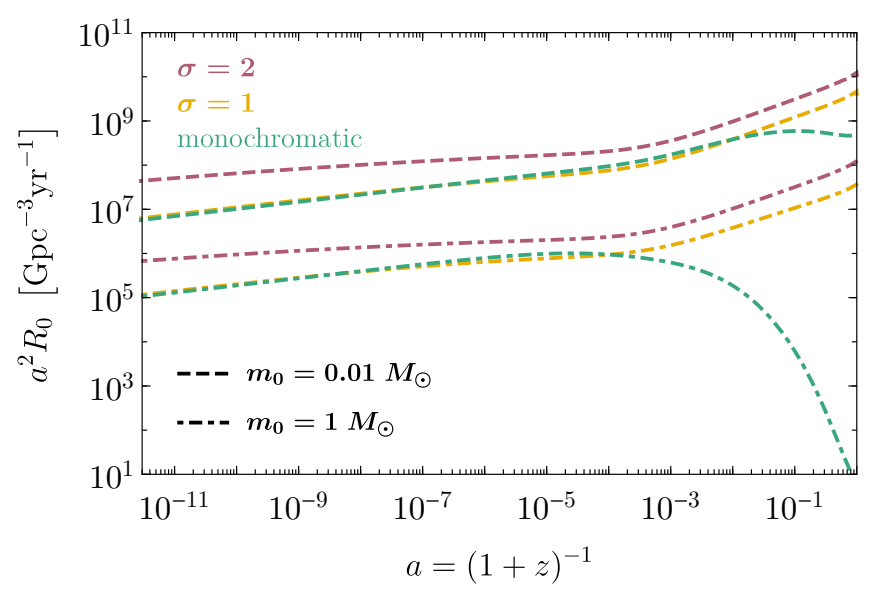

FIG. 4. Evolution of mass-integrated merger rate for lognormal mass distributions with $\sigma=1$ and 2 and monochromatic mass distributions considering only one step with $f_{\mathrm{PBH}}=1$, $\delta_{\mathrm{dc}, 0}=10^{6}$. Note the additional factor of $a^{2}$ on the $y$-axis.

in particular significant changes in the (hierarchy of) the merger rates at the individual steps could potentially entail significant changes in our computations.

Fig. 4 shows the time evolution of the merger rate (integrated over all masses) for different mass distributions for a single merger step, obtained by re-instating an extended mass function in Eq. (6). Here, the coalescence time depends on the masses of the PBHs in the binary $\left(m^{(1)}\right.$ and $\left.m^{(2)}\right)$ and the third PBH $\left(m^{(3)}\right)$ providing angular momentum, and the number density of PBH triples is now given by

$$
\begin{aligned}
\mathrm{d} n_{3,0}^{\log / \mathrm{mono}}(x, y)= & \frac{\rho_{\mathrm{PBH}, \infty}}{2} \mathrm{e}^{-N(y)}\left(4 \pi \rho_{\mathrm{PBH}, \infty} \delta_{\mathrm{dc}, 0}\right)^{2} \\
& x^{2} y^{2} \mathrm{~d} x \mathrm{~d} y \prod_{i=1}^{3} \frac{\psi_{\log / \mathrm{mono}}}{m^{(i)}} \mathrm{d} m^{(i)},
\end{aligned}
$$

where

$$
\begin{aligned}
\rho_{\mathrm{PBH}, \infty}= & \lim _{z \rightarrow \infty}(1+z)^{-3} \rho_{\mathrm{PBH}}(z), \\
N(y)= & \int_{0}^{y} \mathrm{~d} \tilde{y} \int_{0}^{m} \mathrm{~d} \tilde{m} \\
& 4 \pi \tilde{y}^{2} \delta_{\mathrm{dc}, 0} \rho_{\mathrm{PBH}, \infty} \frac{\psi_{\log / \operatorname{mono}}(\tilde{m})}{\tilde{m}}, \\
\psi_{\text {mono }}(m)= & f_{\mathrm{PBH}, \infty} \delta\left(m-m_{0}\right) .
\end{aligned}
$$

For more details see [27. We note that the time evolution of this merger rate does not change significantly when going from a monochromatic to a lognormal mass distribution. This situation only changes at very late times, when the merger rate of this single merger step has however anyway dropped to a small value (note the additional factor of $a^{2}$ in Fig. (4). For the density contrast employed in Fig. 4, $\delta_{\mathrm{dc}, 0}=10^{6}$, subsequent steps start to dominate the total merger rate for $a \gtrsim 5 \cdot 10^{-5}$ and $a \gtrsim 10^{-6}$ for $m_{0}=0.01 M_{\odot}$ and $m_{0}=1 M_{\odot}$, respectively. In conclusion, both the hierarchy among the merger times of the individual merger steps as well as the total amount of mergers are not significantly altered when assuming a rather narrow lognormal mass distribution. For broader distributions the rate is even enhanced.

The SGWB for the lognormal distribution 12 was studied in 27] for a single merger step, finding (for $\sigma=1$ ) a similar GW spectrum as in the monochromatic case. Since the GW radiation from early $\mathrm{PBH}$ mergers experiences a significant redshift, the SGWB in the multiple merger scenario is dominated by the very last merger. Consequently, since we found above that the overall merger history is not significantly altered in the case of a lognormal mass distribution, we conclude that also the resulting SGWB is only moderately altered.

In summary, the strongest constraints depicted in Fig. 3 do not appear to be very sensitive to the assumption of a monochromatic initial PBH mass function. Depending on the mass function in question, they may vary by a factor of a few or even an order of magnitude, but the option of obtaining a significant $\mathrm{PBH}$ dark matter fraction in the LIGO window of $\sim 10 M_{\odot}$ remains firmly excluded. For a given peaked mass distribution one may conservatively estimate the constraints on highly clustered PBH dark matter by considering a narrow window around the central mass value, and applying the analysis of the main text to this window only.

\section{DECREASE OF LOCAL DENSITY CONTRAST}

An important parameter in the modelling of the multiple merger scenario is the decrease in the local density contrast $\delta_{\mathrm{dc}}$, which depends on the details of the two-point correlation function. In a large PBH 'cloud' of constant density, the (number) density contrast stays approximately constant through many merger steps. On the contrary, for PBH 'clouds' in which the typical distance from one PBH binary to the next is enhanced compared to the initial size of the binary, the density contrast drops faster. In the main text, we considered $\delta_{\mathrm{dc}, \mathrm{j}}=0.5^{j} \delta_{\mathrm{dc}, 0}$, which we consider a conservative approach for highly clustered PBH distributions.

Here we also discuss the case of a locally approximately homogeneous density contrast. To model this, we consider $\delta_{\mathrm{dc}, \mathrm{i}}=0.9^{j} \delta_{\mathrm{dc}, 0}$. The resulting constraints, depicted in Fig. 5 for $\delta_{\mathrm{dc}, 0}=10^{3}$, are clearly significantly tighter than those presented in the main text. Note that in this case the bounds can be extrapolated down to very low masses. Taken at face value, this even allows to constrain masses as low as $m_{0}=10^{-16} M_{\odot}$, but this extrapolation implicitly assumes very large overdensities of $\mathcal{O}\left(10^{15}\right) \mathrm{PBHs}$, which seems hardly realistic. We also note that such a slow decrease in the density contrast weakens the hierarchy between the merger times of the 


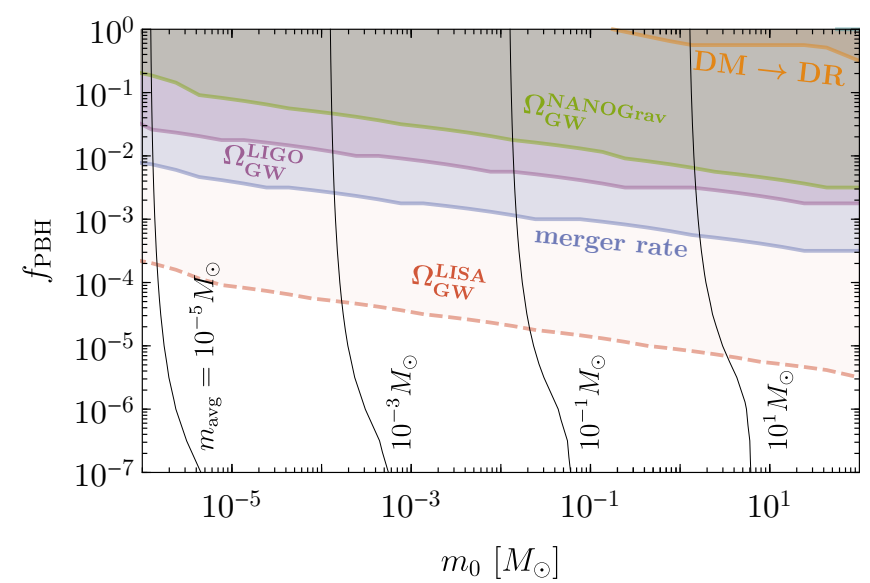

FIG. 5. Constraints for smaller initial clustering, $\delta_{\mathrm{dc}, 0}=10^{3}$ and $\delta_{\mathrm{dc}, \mathrm{j}}=0.9^{j} \delta_{\mathrm{dc}, 0}$. See Fig. 3 for details.

individual merger steps, making our analysis less robust.

We conclude this appendix by noting that for this slower decrease in the density contrast corresponding to rather homogeneous $\mathrm{PBH}$ 'clouds', the formation of $N$ body systems may lead to a more complicated merger history not covered by the treatment in this letter 62.

\section{GRAVITATIONAL WAVE RECOIL}

Due to asymmetries in the emitted gravitational radiation, the remnant of merging binary $\mathrm{BHs}$ can gain recoil velocities of $\mathcal{O}(100-1000 \mathrm{~km} / \mathrm{s})$ 63 65]. These velocities are zero in the equal-mass zero-spin case, but differ from zero if either of these conditions are not met. Specifically, a maximal velocity of $\sim 175 \mathrm{~km} / \mathrm{s}$ can be obtained in the zero-spin case for a $\mathrm{BH}$ mass ratio of 0.36 . For equal masses and non-zero spins with special alignment the maximum recoil velocity can be as large as $\sim 5000 \mathrm{~km} / \mathrm{s}$ 65. As special spin alignment is unlikely in the context of this work, we expect recoil velocities of $\mathcal{O}(100 \mathrm{~km} / \mathrm{s})$. In our treatment of multiple merger steps we have implicitly assumed that recoils do not affect the evolution of the PBHs. Here we show that this approximation is justified, by comparing these recoil velocities to the relevant escape velocities.

The escape velocity from a PBH cluster will depend on its global structure and hence on the two-point correlation function. As we take the local density contrast to decrease as $\delta_{\mathrm{dc}, j} \simeq 2^{-j} \delta_{\mathrm{dc}, 0}$, we expect initial clusters to contain $\sim \delta_{\mathrm{dc}, 0} \mathrm{PBHs}$ with comoving radius $x_{\text {clus }}=\left(3 / 4 \pi n_{0}\right)^{1 / 3}$, such that $4 \pi x_{\text {clus }}^{3} n_{0} \delta_{\mathrm{dc}, 0} / 3=\delta_{\mathrm{dc}, 0}$. These clusters decouple from the Hubble flow during radiation domination when the local energy density in PBHs $\rho_{\mathrm{PBH}} \delta_{\mathrm{dc}, 0}$ is equal to the background density. This gives a decoupling scale factor and an escape velocity from the cluster of

$$
\begin{aligned}
a_{\mathrm{dc}, \text { clus }} & =a_{\mathrm{eq}}^{4} \frac{\rho_{\mathrm{eq}}}{2 m_{0} n_{0} \delta_{\mathrm{dc}, 0}} \\
v_{\mathrm{esc}, \text { clus }} & \simeq \sqrt{\frac{2 G \delta_{\mathrm{dc}, 0} m_{0}}{x_{\mathrm{clus}} a_{\mathrm{dc}, \mathrm{clus}}}} \\
& =3600 \frac{\mathrm{km}}{\mathrm{s}} f_{\mathrm{PBH}}^{2 / 3}\left(\frac{m_{0}}{M_{\odot}}\right)^{1 / 3} \frac{\delta_{\mathrm{dc}, 0}}{10^{4}} .
\end{aligned}
$$

This implies that for most regions of parameter space recoils should not affect our results.

This conclusion may seemingly be challenged for small initial masses and small values of $f_{\mathrm{PBH}}$. In this case, however, our constraints are driven by rare events of many merger steps, implying that initially the PBHs were exceptionally close. Specifically note that any mergers we take into account in our limits must have a coalescence time $\tau$ smaller than the age of the universe $t_{0}$, i.e.

$$
\tau=\tilde{\tau}_{j}\left(x / \tilde{x}_{j}\right)^{37}\left(y / \tilde{x}_{j}\right)^{-21} \leq t_{0} .
$$

Conservatively estimating $y \simeq 2^{1 / 3} x$ (smaller values give larger escape velocities and larger values are exponentially suppressed by the exponential term in Eq. (7)), the escape velocity from a binary $\mathrm{BH}$ in merger step $j$ can be written as

$$
v_{\text {esc }, \text { bin }}=\sqrt{\frac{2 G m_{j}}{x a_{\mathrm{dc}, j}}} \gtrsim 190 \frac{\mathrm{km}}{\mathrm{s}}\left(\frac{m_{j}}{M_{\odot}}\right)^{1 / 8}
$$

where $a_{\mathrm{dc}, j}$ is the scale factor at which the binary system decouples from the Hubble flow. We see that PBHs with $m_{j} \gtrsim 0.1 M_{\odot}$, which are relevant for the merger rate and the stochastic GW background from LIGO, typically do not pick up a sufficient recoil in their previous merger to escape the gravitational attraction of even their nearest neighbour. Note however that even sub-critical recoils will generally lead to dynamical heating. A detailed analysis of this effect is beyond the scope of this work.

In summary, due to the early formation time, the relevant $\mathrm{PBH}$ escape velocities are very large in the highly clustered scenarios we have studied here, allowing to neglect the effect of GW recoils in our analysis.

* torsten.bringmann@fys.uio.no

$\dagger$ frederik.depta@desy.de

$\ddagger$ valerie.domcke@desy.de

$\S$ kai.schmidt-hoberg@desy.de

[1] Y. B. Zel'dovich and I. D. Novikov, Sov. Astron. 10, 602 (1967).

[2] S. Hawking, Mon. Not. Roy. Astron. Soc. 152, 75 (1971).

[3] B. J. Carr and S. W. Hawking, Mon. Not. Roy. Astron. Soc. 168, 399 (1974).

[4] G. F. Chapline, Nature 253, 251 (1975) 
[5] B. Carr, F. Kuhnel, and M. Sandstad, Phys. Rev. D94, 083504 (2016), arXiv:1607.06077 [astro-ph.CO]

[6] M. Sasaki, T. Suyama, T. Tanaka, and S. Yokoyama, Class. Quant. Grav. 35, 063001 (2018), arXiv:1801.05235 [astro-ph.CO].

[7] S. Bird, I. Cholis, J. B. Muñoz, Y. Ali-Haïmoud, M. Kamionkowski, E. D. Kovetz, A. Raccanelli, and A. G. Riess, Phys. Rev. Lett. 116, 201301 (2016), arXiv:1603.00464 [astro-ph.CO].

[8] S. Clesse and J. García-Bellido, Phys. Dark Univ. 15, 142 (2017), arXiv:1603.05234 [astro-ph.CO]

[9] M. Sasaki, T. Suyama, T. Tanaka, and S. Yokoyama, Phys. Rev. Lett. 117, 061101 (2016), [Erratum: Phys. Rev. Lett.121,no.5,059901(2018)], arXiv:1603.08338 [astro-ph.CO].

[10] B. Carr, M. Raidal, T. Tenkanen, V. Vaskonen, and H. Veermäe, Phys. Rev. D96, 023514 (2017). arXiv:1705.05567 [astro-ph.CO].

[11] S. Wang, Y.-F. Wang, Q.-G. Huang, and T. G. F. Li, Phys. Rev. Lett. 120, 191102 (2018), arXiv:1610.08725 [astro-ph.CO],

[12] B. P. Abbott et al. (Virgo, LIGO Scientific), Phys. Rev. Lett. 116, 061102 (2016), arXiv:1602.03837 [gr-qc]

[13] B. P. Abbott et al. (Virgo, LIGO Scientific), Phys. Rev. Lett. 116, 241103 (2016), arXiv:1606.04855 [gr-qc]

[14] B. P. Abbott et al. (Virgo, LIGO Scientific), Phys. Rev. X6, 041015 (2016), arXiv:1606.04856 [gr-qc]

[15] B. P. Abbott et al. (VIRGO, LIGO Scientific), Phys. Rev. Lett. 118, 221101 (2017), arXiv:1706.01812 [gr-qc]

[16] J. García-Bellido and S. Clesse, Phys. Dark Univ. 19, 144 (2018), arXiv:1710.04694 [astro-ph.CO]

[17] M. Zumalacarregui and U. Seljak, arXiv:1712.02240 [astro-ph.CO],

[18] J. Garcia-Bellido, S. Clesse, and P. Fleury, Phys. Dark Univ. 20, 95 (2018), arXiv:1712.06574 [astro-ph.CO]

[19] J. R. Chisholm, Phys. Rev. D73, 083504 (2006), arXiv:astro-ph/0509141 [astro-ph]

[20] J. R. Chisholm, Phys. Rev. D84, 124031 (2011) arXiv:1110.4402 [astro-ph.CO],

[21] V. Dokuchaev, Y. Eroshenko, and S. Rubin, Grav. Cosmol. 11, 99 (2005), arXiv:astro-ph/0412418 [astro-ph].

[22] V. I. Dokuchaev, Yu. N. Eroshenko, and S. G. Rubin, (2007), arXiv:0709.0070 [astro-ph]

[23] Y. Ali-Haïmoud, Phys. Rev. Lett. 121, 081304 (2018) arXiv:1805.05912 [astro-ph.CO]

[24] V. Desjacques and A. Riotto, (2018), arXiv:1806.10414 [astro-ph.CO].

[25] S. Young and C. T. Byrnes, Phys. Rev. D91, 083521 (2015), arXiv:1411.4620 [astro-ph.CO]

[26] K. M. Belotsky, V. I. Dokuchaev, Y. N. Eroshenko, E. A. Esipova, M. Yu. Khlopov, L. A. Khromykh, A. A. Kirillov, V. V. Nikulin, S. G. Rubin, and I. V. Svadkovsky, (2018), arXiv:1807.06590 [astro-ph.CO]

[27] M. Raidal, V. Vaskonen, and H. Veermäe, JCAP 1709, 037 (2017), arXiv:1707.01480 [astro-ph.CO]

[28] G. Ballesteros, P. D. Serpico, and M. Taoso, (2018), arXiv:1807.02084 [astro-ph.CO],

[29] B. Carr and J. Silk, (2018), 10.1093/mnras/sty1204, arXiv:1801.00672 [astro-ph.CO]

[30] N. Aghanim et al. (Planck), (2018), arXiv:1807.06209 [astro-ph.CO].

[31] See Supplemental Material at [URL will be inserted by publisher].

[32] M. Maggiore, Gravitational Waves. Vol. 2: Astrophysics and Cosmology (Oxford University Press, 2018).

[33] B. P. Abbott et al. (LIGO Scientific, Virgo), (2018), arXiv:1811.12907 [astro-ph.HE].

[34] E. S. Phinney, (2001), arXiv:astro-ph/0108028 [astro$\mathrm{ph}]$.

[35] B. P. Abbott et al. (Virgo, LIGO Scientific), Phys. Rev. Lett. 116, 131102 (2016), arXiv:1602.03847 [gr-qc]

[36] T. Bringmann, F. Kahlhoefer, K. Schmidt-Hoberg, and P. Walia, Phys. Rev. D98, 023543 (2018), arXiv:1803.03644 [astro-ph.CO]

[37] C. Cutler, E. Poisson, G. J. Sussman, and L. S. Finn, Phys. Rev. D47, 1511 (1993)

[38] X.-J. Zhu, E. Howell, T. Regimbau, D. Blair, and Z.H. Zhu, Astrophys. J. 739, 86 (2011), arXiv:1104.3565 [gr-qc].

[39] D. F. Chernoff and L. S. Finn, Astrophys. J. 411, L5 (1993), arXiv:gr-qc/9304020 [gr-qc].

[40] P. Ajith et al., Phys. Rev. D77, 104017 (2008), [Erratum: Phys. Rev.D79,129901(2009)], arXiv:0710.2335 [gr-qc].

[41] T. Nakamura, M. Sasaki, T. Tanaka, and K. S. Thorne, Astrophys. J. 487, L139 (1997), arXiv:astro-ph/9708060 [astro-ph]

[42] K. Ioka, T. Chiba, T. Tanaka, and T. Nakamura, Phys. Rev. D58, 063003 (1998), arXiv:astro-ph/9807018 [astro$\mathrm{ph}]$

[43] G. D. Quinlan and S. L. Shapiro, Astrophys. J. 343, 725 (1989).

[44] H. Mouri and Y. Taniguchi, Astrophys. J. 566, L17 (2002), arXiv:astro-ph/0201102 [astro-ph]

[45] Y. Ali-Haïmoud, E. D. Kovetz, and M. Kamionkowski, Phys. Rev. D96, 123523 (2017), arXiv:1709.06576 [astroph.CO].

[46] P. C. Peters, Phys. Rev. 136, B1224 (1964)

[47] V. Poulin, P. D. Serpico, and J. Lesgourgues, JCAP 1608, 036 (2016), arXiv:1606.02073 [astro-ph.CO]

[48] Z. Arzoumanian et al. (NANOGRAV), Astrophys. J. 859, 47 (2018), arXiv:1801.02617 [astro-ph.HE].

[49] H. Audley et al. (LISA), (2017), arXiv:1702.00786 [astroph.IM]

[50] B. P. Abbott et al. (Virgo, LIGO Scientific), Phys. Rev. Lett. 118, 121101 (2017), [Erratum: Phys. Rev. Lett.119,no.2,029901(2017)], arXiv:1612.02029 [gr-qc]

[51] M. Maggiore, Gravitational Waves. Vol. 1: Theory and Experiments, Oxford Master Series in Physics (Oxford University Press, 2007).

[52] B. P. Abbott et al. (Virgo, LIGO Scientific), Astrophys. J. 833, L1 (2016), arXiv:1602.03842 [astro-ph.HE],

[53] B. P. Abbott et al. (Virgo, LIGO Scientific), Astrophys. J. Suppl. 227, 14 (2016), arXiv:1606.03939 [astroph.HE]

[54] B. P. Abbott et al. (Virgo, LIGO Scientific), Phys. Rev. D96, 022001 (2017), arXiv:1704.04628 [gr-qc].

[55] B. P. Abbott et al. (Virgo, LIGO Scientific), (2018), arXiv:1808.04771 [astro-ph.CO]

[56] H. Niikura et al., (2017), arXiv:1701.02151 [astroph.CO]

[57] B. J. Carr, K. Kohri, Y. Sendouda, and J. Yokoyama, Phys. Rev. D81, 104019 (2010), arXiv:0912.5297 [astroph.CO]

[58] A. A. Starobinsky, JETP Lett. 55, 489 (1992), [Pisma Zh. Eksp. Teor. Fiz.55,477(1992)].

[59] D. Blais, T. Bringmann, C. Kiefer, and D. Polarski, Phys. Rev. D67, 024024 (2003), arXiv:astro-ph/0206262 [astro-ph] 
[60] D. Blais, C. Kiefer, and D. Polarski, Phys. Lett. B535, 11 (2002), arXiv:astro-ph/0203520 [astro-ph]

[61] N. Orlofsky, A. Pierce, and J. D. Wells, Phys. Rev. D95, 063518 (2017), arXiv:1612.05279 [astro-ph.CO]

[62] M. Raidal, C. Spethmann, V. Vaskonen, and H. Veermäe, Submitted to: JCAP (2018), arXiv:1812.01930 [astro-ph.CO],

[63] M. Koppitz, D. Pollney, C. Reisswig, L. Rezzolla,
J. Thornburg, P. Diener, and E. Schnetter, Phys. Rev. Lett. 99, 041102 (2007), arXiv:gr-qc/0701163 [GR-QC]

[64] L. Rezzolla, E. N. Dorband, C. Reisswig, P. Diener, D. Pollney, E. Schnetter, and B. Szilagyi, Astrophys. J. 679, 1422 (2008), arXiv:0708.3999 [gr-qc]

[65] C. O. Lousto and Y. Zlochower, Physical Review Letters 107, 231102 (2011), arXiv:1108.2009 [gr-qc]. 